\title{
Self-consistent Computations of the Geometries of Magnetically Confined Liquid Metal Columns
}

\author{
David W. FUGATE and James F. HOBURG \\ Department of Electrical and Computer Engineering, Carnegie-Mellon University, Pittsburgh, PA 15213, U.S.A.
}

(Received on February 13, 1989; accepted in the final form on May 19, 1989)

\begin{abstract}
In this paper, a modeling methodology is described which permits the computation of the cross-sectional geometry of a two dimensional column of perfectly conducting liquid in the presence of a magnetic field imposed by specified configurations of source conductors. The description is self-consistent in the sense that the column geometry results from a magnetic pressure corresponding to a magnetic field determined by the column geometry. Solutions are obtained by iteratively calculating the magnetic field structure for a given column geometry and source conductors, and then calculating a new column geometry based on the magnetic stresses, surface tension, and pressure jump at the liquid-air interface. This loop is continued until a self-consistent solution is obtained. A quadrupole source magnetic shaping problem solved by Shercliff using conformal mapping is duplicated using the methodology described in this paper. The results obtained are in good agreement with those published by Shercliff. In addition, results are shown for arbitrary shaping problems, including levitation of horizontal columns, that are too complex for analytic solutions.
\end{abstract}

KEY WORDS: magnetic confinement; magnetic levitation; liquid metals.

\section{Introduction}

Containment of the liquid phase is a basic and continuing problem in the casting of metals. In steelmaking, for example, containers have a limited life, extended downtime between uses, and are susceptible to premature failure. Refractory materials generally experience a very corrosive environment and are subjected to extreme friction forces. In modern developments, where a near-net-shape product is cast, nozzle material requirements are so severe that no suitable refractory materials currently exist. ${ }^{11}$

Problems of the kind described here have motivated considerable interest in the potential for using magnetic fields to confine liquid metals. There have been a number of studies of magnetic levitation and melting as a means to prepare laboratory specimens of highly reactive metals and alloys such as titanium, zirconium and aluminum-killed steels. ${ }^{2-4)}$ Magnetic containment and shaping of liquid metals has the potential to alleviate some of the materials problems facing the development of strip casting.

The work described in this paper provides a means for determining the interfacial geometry of a column of liquid metal which experiences a "magnetic pressure" due to induced currents at its surface in the presence of a specified source coil configuration. Since the geometry of the column affects the magnetic field structure, the electromechanical coupling is two-way; the field determines the geometry and the geometry determines the field. A self-consistent determination of both interfacial geometry and magnetic field is necessary.

Shercliff has obtained self-consistent solutions of ellipse-like and four-cusped hypocycloid liquid column shapes resulting from uniform far field and four conductor quadrupole fields respectively, using conformal mapping coupled with numerical integration. ${ }^{51}$ However, Shercliff's method cannot be straightforwardly generalized to different source coil configurations. More recently, Gagnoud et al. have solved for the equilibrium shape of liquid metal levitated and shaped by axisymmetric conical coils using iterative local and global methods for solving the non-linear coupling between magnetic field and liquid metal free boundary. ${ }^{6}$ In the local method the free boundary shape is solved using a Green's function-integral equation technique. In the global method, the free boundary shape is found by minimizing the energy functional of the system.

The work described here employs a second order finite element method with automatic mesh generation to calculate magnetic field structure for a previously calculated interface geometry and the stress balance equation at the air-liquid interface to calculate a new interface geometry based on the previously calculated magnetic field and corresponding magnetic pressure distribution. Iterative use of these two methods converges to a self-consistent solution for general source coil configurations.

\section{Simplifying Assumptions and Nature of the Model}

The model developed here incorporates several simplifying assumptions:

(1) The liquid metal column geometry is twodimensional. Variations of column geometry in the 
direction of the column axis are assumed to occur slowly enough that they can be neglected in calculating the cross-sectional shape. The validity of this assumption is highly dependent upon the specific magnetic confinement application to which the description is applied.

(2) The liquid metal column is treated as a perfect conductor in determining its effect upon the magnetic field. That is, the frequency of the applied alternating magnetic field is assumed to be large enough to justify approximating the skin depth as zero. The electromechanical description is thus surface-coupled.

This approximation is likely to be relatively poor at practical frequencies. For example, a frequency of $3500 \mathrm{~Hz}$ results in a skin depth of $10 \mathrm{~mm}$ in liquid steel at $1530^{\circ} \mathrm{C}$. When the magnetic field penetrates a significant distance into the liquid metal, a rotational Lorentz force density can give rise to bulkcoupled magnetohydrodynamic motions. This sort of interaction is neglected in the model described here.

(3) The magnetic surface force density, which has a constant part and a part which alternates at twice the frequency of the imposed magnetic field, is approximated by only the constant part. Thus, the source frequency is assumed to be large enough that the fluid cannot mechanically respond to the alternating part of the force density. Due to viscosity and inertia of the liquid metal, this approximation is well satisfied at practical operating frequencies.

The model described here provides a description of the liquid metal column geometry for a general configuration of source currents, and thus permits experimentation with source distribution and resultant column shape. It is important to distinguish this sort of description from a straightforward computation of magnetic field distribution based upon a desired column geometry. Because the metal column is a fluid, its geometry cannot simply be specified and the necessary field then determined. Such a calculation of field structure based upon assumed column geometry would involve an implicit imbalance in surface force densities. The description must be self-consistent, in the sense that the static fluid geometry must satisfy the surface stress balance at the same time it determines the magnetic field distribution which produces the magnetic stress.

Chapters 3 and 4 describe specific means for achieving quantitative descriptions of both links in the electromechanical coupling. Each link assumes a known drive, as supplied by a prior calculation of the other link. The overall method is based upon iterative calculation, converging to a self-consistent solution.

\section{Calculation of Magnetic Field for Known Interface Geometry}

In this part of the iterative procedure, the objective is calculation of the magnetic field structure, and thus magnetic stress distribution, for given source conductors and a given air-liquid interface geometry. Like the induced currents in the liquid metal, the source currents are assumed to be confined to the outer surface of the solid conductor coils; the magnetic field is purely tangential at these surfaces. The magnetic field structure can be found by solving for the magnetic vector potential that satisfies Laplace's equation, subject to the boundary condition of constant magnetic vector potentials along the "perfectly conducting" surfaces, in the insulating air region. The potential difference specifies the magnetic flux per unit length between the source conductors and liquid metal.

Conductor coils composed of many insulated wires can also be modeled by specifying a uniform current density over the coil cross section, and then finding a vector potential solution that satisfies Poisson's equation over the entire problem region. In this case, there is still a constant magnetic vector potential boundary condition on the liquid metal column.

The magnetic field is obtained from the magnetic vector potential solution. Because the magnetic field is excluded from the liquid metal and the solid source conductors, the surface currents are equal in magnitude to the magnetic field intensity at the surfaces. The total current can be found using Ampere's Law by integrating a closed path around each conductor. In principle, any numerical method with the capability to solve the proper boundary value problem for arbitrary geometries can be used in this part of the iterative procedure to calculate the magnetic stress distribution.

The finite element method for solution of the partial differential equations governing quasistatic magnetic fields with known sources and "perfectly" conducting boundaries is well established. ${ }^{7-11)}$ In order to handle a column boundary whose position is the result of a prior calculation, a means for automatically generating a finite element mesh during each iteration is desirable. The model described here incorporates preexisting finite element procedures which involve Delaunay triangulation as means for logically and efficiently creating a finite element mesh for a calculated column surface geometry. ${ }^{12)}$ The Delaunay procedure takes as input a set of finite element nodes on boundaries and within the bulk of a region where a finite clement solution is desired. It produces as output a triangulation of the region, with all triangle vertices lying on the specified nodes. This procedure maximizes the sum over all triangles of the smallest angle in each. Because finite element solutions are most accurate for nearly equilateral triangles, Delaunay triangulation provides a mesh for the finite element procedure which produces generally excellent numerical results.

Finally, the magnetic stress acting on the liquid metal at each point along the boundary is given by the magnetic stress tensor $T_{n n}$ derived from the Lorentz force equation:

$$
T_{n n}=-\frac{1}{2} \mu_{0} H_{t}^{2}
$$

where $\mu_{0}$ is the permeability of free space, and $H_{t}$ is 
the magnetic field intensity; the subscript represents the fact that the field has no normal component.

In summary, the mesh procedure takes the data representing the column boundary and creates a new mesh. This new mesh is used by the finite element procedure to calculate a new magnetic field structure. The magnetic stress distribution obtained from $\mathrm{Eq}$. (1) is the data necessary for the second part of the iterative procedure. To start the iterative procedure, the initial column shape which specifies the cross-sectional area of liquid metal, the source coil geometries, and additional mesh refinement points are specified using interactive computer graphics.

\section{Calculation of Column Geometry for Known Magnetic Stress Distribution}

Stress equilibrium at the interface between the metal column and the surrounding air or gaseous environment requires a balance between the surface force density resulting from the induced surface current density and the magnetic field just outside the column, surface tension, and pressure jump across the interface. The stress balance equation is:

$$
\|p\|+\gamma(\nabla \cdot \vec{n})-T_{n n}=0
$$

where $\gamma(\nabla \cdot \vec{n})$, the divergence of the normal to the interface times the surface tension constant $\gamma$, is the stress contribution due to surface tension, $\|p\|$ is the pressure jump, $p_{o u}-p_{\mathrm{in}}$ at the interface, and $T_{n n}$ is the known magnetic surface force density.

By representing the interface in polar coordinates, the radius as a function of the angular position $\theta$, finding the analytical expression for the divergence of the normal, and substituting this expression into the stress balance equation, one obtains a second order nonlinear differential equation. This equation may be solved using numerical integration to obtain a new column geometry. The derivation of the nonlinear differential equation and two such schemes to solve it are described in more detail in Ref. 13).

Another technique for calculating a new column geometry based on the stress distribution is free movement relaxation. In this method, the boundary that represents the column geometry is allowed to move in or out (based on the stress imbalance) on each iteration until an equilibrium shape is reached. Though not as elegant as numerical solution of the nonlinear differential equation that was derived from Eq. (2), this method is much more robust. While convergence problems are encountered with the numerical integration schemes, the free movement method has the capability to model a column geometry at the high pressures necessary to confine and levitate steel.

In the free movement relaxation method, the liquid column boundary is represented by a set of points connected by line segments. After solving for the magnetic field structure, the magnetic stresses are known at each point on the boundary. Movement of the points based upon the local stress imbalance at each point gives the new column geometry for the next iteration.
Because the free boundary that defines the liquid metal column is represented by a series of points connected by line segments, it is necessary to derive a discrete version of surface tension. One way of accomplishing this is to look at an equilibrium of the stresses along one small section of the free boundary (a point and one half of the adjacent line segments). Each point on the boundary is the vertex of an angle formed by the two line segments connecting the adjacent points. We can define the normal vector to the boundary as the bisector of this angle, as shown in Fig. 1. This is the average of the two outward normal vectors of the two adjacent line segments. If surface tensions, acting along the two line segments in opposite directions, exactly balance some pressure jump $P$ (which is actually a combination of hydrostatic and magnetic pressures) then

$$
P d=2 \gamma \sin \theta,
$$

where $d$ is the distance shown in Fig. $1, \gamma$ is the surface tension constant, and $\theta$ is the angle between the average normal vector and the normal vector of either of the adjacent line segmenis. Due to symmetry (line segments are of equal length), stresses cancel in the tangential direction and $\mathrm{Eq}$. (3) represents a balance of stresses in the normal direction. The $\sin \theta$ term picks off the normal components of surface tension which balance $P d$, the stress in the normal direction due to the pressure jump and magnetic stress at the interface. Solving for $P$ we obtain

$$
P=\frac{2}{d} \gamma \sin \theta
$$

which gives us a discrete measure of the stress contribution due to surface tension. This is similar to the well known equation $P=\gamma / R$ where $R$ is the local radius of curvature. The term $(2 / d) \sin \theta$ is a discrete measure of $1 / R$, the inverse of the local radius of curvature. The stress balance is now given by Eq. (5).

$$
\|p\|+\frac{2}{d} r \sin \theta-\mathcal{T}_{n n}=0
$$

While the surface tension and magnetic stresses are known, the pressure jump at the interface is a free variable that is determined by the effects of the magnetic stresses, surface tension, and gravity. Because the pressure jump along the boundary is not known a priori, the highest point on the column boundary is

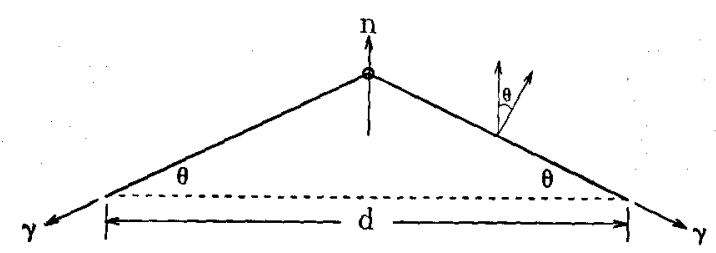

Fig. 1. One section of the discretized free boundary, a boundary point and one half of the adjacent line segments that connect this point to the adjacent boundary points. This basic section of the free boundary is used to derive a discrete model of the stress contribution due to surface tension. 
assigned a pressure jump of zero. All other points are assigned a pressure equal to the hydrostatic head term $\rho g h$, where $\rho$ is the density of the liquid, $g$ is gravitational acceleration, and $h$ is the difference in elevation between a point and the highest point of the liquid metal. Movement of the boundary points (in a direction normal to the boundary) is based on the average stress imbalance. Points with a stress imbalance greater than the average are moved outward and points with a stress balance less than the average are moved inward. The distance each point is moved should be small with respect to dimensions of the liquid metal column to stabilize the iterative procedure.

In order to give equal "weight" to all parts of the column geometry, the points are spaced equally along the boundary after each iteration. Area is conserved on each iteration by matching the increase of column area due to points moving outward a distance $d_{\text {out }}$ with the decrease of area due to points moving inward a distance $d_{\mathrm{in}}$. Using this method in the iterative procedure, the column geometry relaxes into an equilibrium where all points see the same stress imbalance. This stress imbalance is the pressure jump at the highest point on the column boundary and can be added to the hydrostatic head term at the other points along the column boundary to obtain the actual pressure jump.

Chapter 5 describes results obtained using the finite element procedure to calculate a magnetic stress distribution and the free movement model to calculate a column geometry as the two parts of an iterative procedure that results in self-consistent solutions.

\section{Results}

In order to verify the results of the iterative procedure described here, a comparison has been made with the results of Shercliff ${ }^{5}$ based upon a conformal mapping for a symmetric problem involving shaping of a vertical liquid metal column by a quadrupolelike magnetic field produced by a source configuration of four vertical conductors.

Fig. 2 shows a finite element mesh generated by the Delaunay algorithm for the Shercliff problem. A quadrupole-like field is produced by the four wire conductors with currents in top and bottom wires opposite from the currents in the side wires. Fig. 3 shows a comparison of one of Shercliff's solutions with a solution from the gencral purpose model described here. The curves describe one-quarter of the symmetric interface geometry. The boundary was discretized into 48 points.

The parameter " $a$ ", set to 1000 in the results shown in Fig. 3 is Shercliff's dimensionless measure of the magnetic stress relative to the pressure jump across the interface. Excellent agreement has been obtained in all comparisons with Shercliff's conformal mapped results. Fig. 4 shows a series of full solutions from $a=0$ (a perfect circle) to $a=1000$.

Fig. 5 shows the self-consistent magnetic flux density and interface geometry for a horizontal column

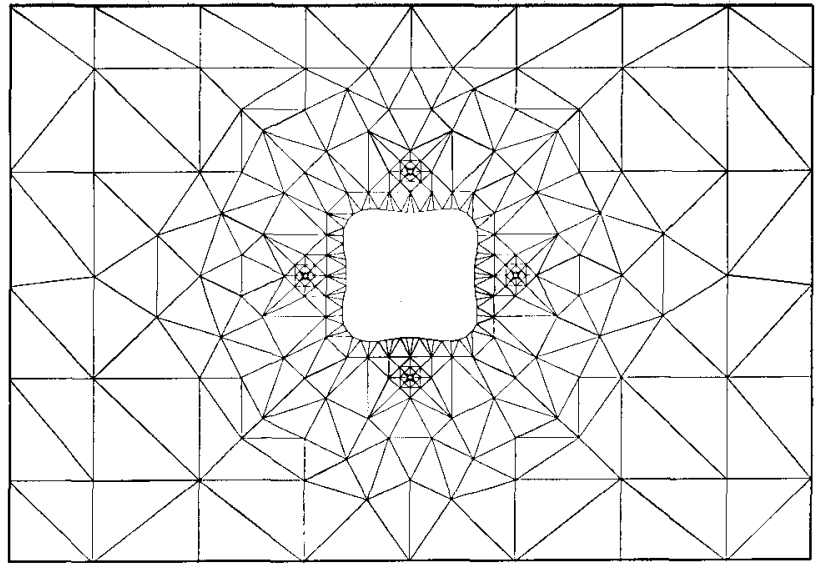

Fig. 2. A finite element mesh for the problem posed by Shercliff, where the horizontal magnetic field produced by four vertical wire conductors shapes a vertical liquid metal column.

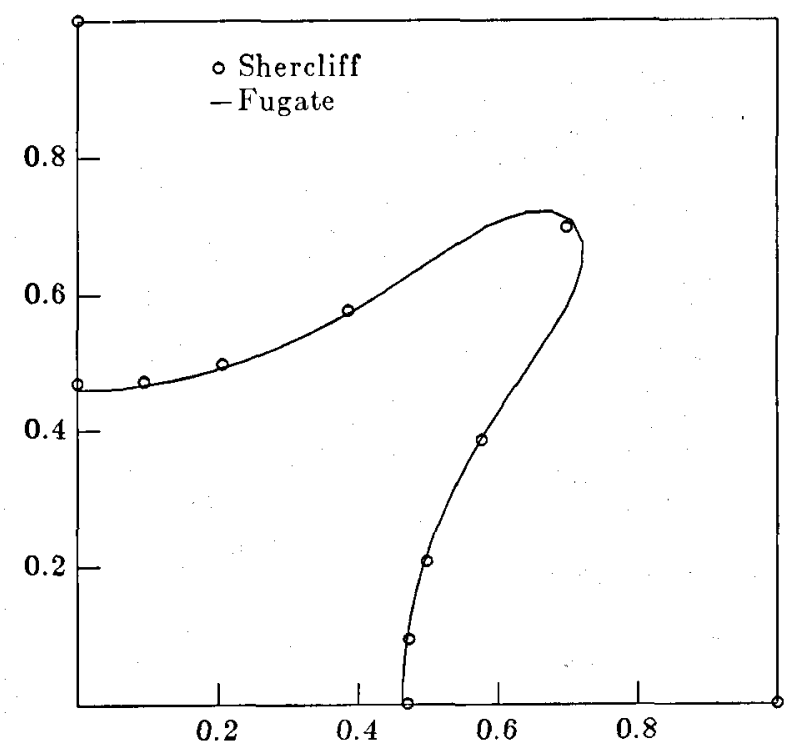

Fig. 3. Comparison of a self-consistent solution (one quarter) with one of Shercliff's conformal mapped solutions for $a=1000$, where $a$ is Shercliff's dimensionless measure of magnetic stress relative to pressure jump across the interface.

of liquid metal levitated by a solid conductor. In this example, the difference in magnctic vector potential between the conductor coil and the liquid metal determines the flux density per unit length between the two objects. The liquid metal moves toward the coil, compressing the flux until the magnetic field is strong enough to levitate the liquid metal. The column is approximately $40 \mathrm{~mm}$ by $180 \mathrm{~mm}$. The liquid metal has a density of $7000 \mathrm{~kg} / \mathrm{m}^{3}$ and a surface tension constant of $1 \mathrm{~N} / \mathrm{m}$. A mesh of 230 triangles was used in the magnetic field calculations and the boundary was discretized into 68 points.

Finally, Fig. 6 shows a series of three interface geometries that correspond to self-consistent solutions for a system with a solid horizontal conductor that wraps back over the liquid metal. The conductor is 320 $\mathrm{mm}$ by $160 \mathrm{~mm}$. The liquid metal surface tension constant and density are the same as for the previous 


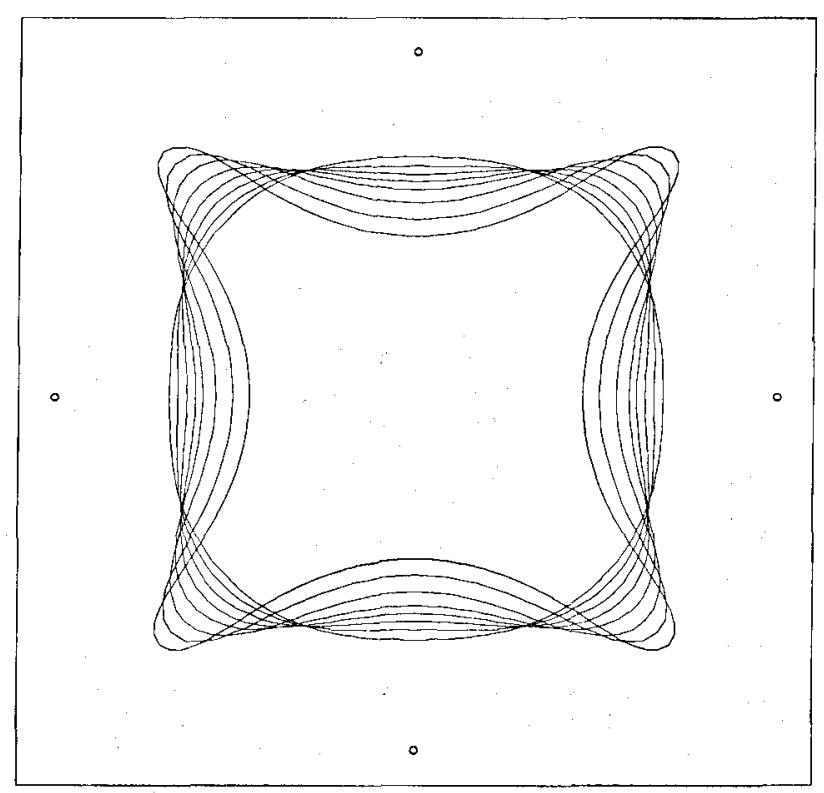

Fig. 4. A series of vertical column geometries that are solutions to Shercliff's four vertical conductor problem. The solutions correspond to increasing values of $a$, Shercliff's dimensionless measure of magnetic stress relative to pressure jump across the interface.

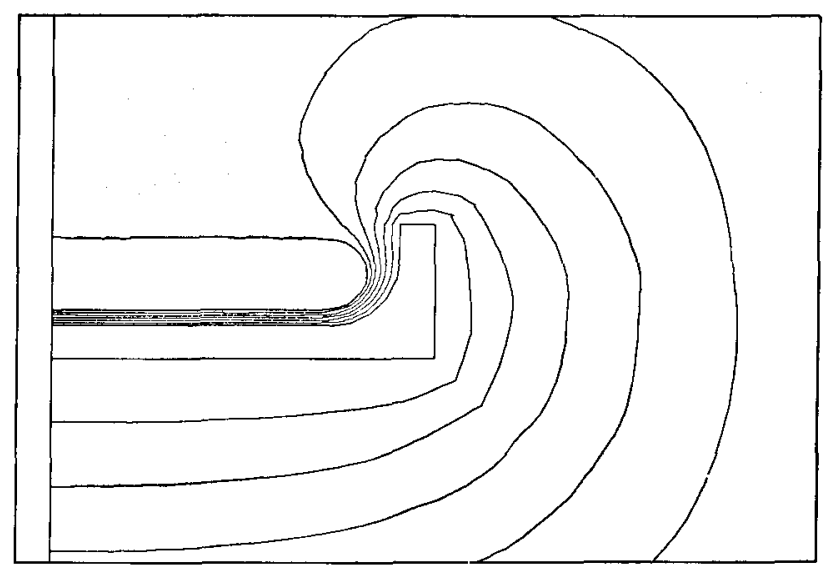

Fig. 5. One half of a self-consistent magnetic field structure and horizontally levitated liquid metal column geometry.

solution. As the flux between the conductor and liquid metal increases, the liquid is squeezed up into the open region. The cross-sectional area has been maintained from solution to solution. The finite element mesh consists of 250 triangles and the boundary is discretized into 32 points for this problem. More boundary points can be added to increase the accuracy of the column geometry in the regions of highest curvature.

The number of iterations necessary for convergence to a self-consistent solution depends on the problem geometry, initial column boundary, and distance the points are moved on each iteration. The solutions shown in this section required on the order of 100 iterations. On a $\mu$ VAX II, each iteration requires about $240 \mathrm{~s}$ of GPU time. The mesh procedure, the finite element solver, and the free movement procedure were run iteratively using a Unix C-shell pro-

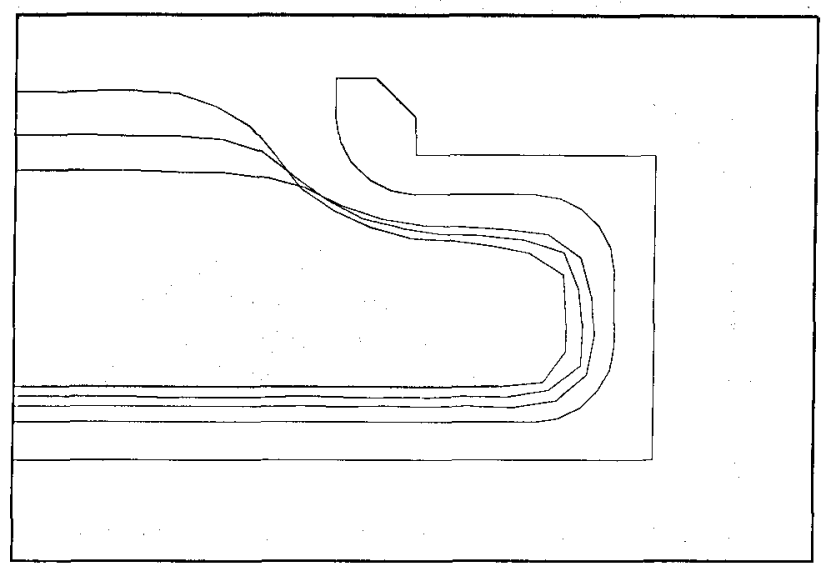

Fig. 6. A series of self-consistent interface geometries corresponding to increasing flux between the horizontal inductor and horizontal liquid metal column.

gram. The GPU time per iteration could be significantly reduced by customizing the finite element procedures and streamlining all three programs into a single program.

\section{Conclusions}

The modeling methodology described in this paper provides, for the first time, a general purpose tool for determination of self-consistent two dimensional liquid conductor geometries which result from magnetic fields imposed by specified configurations of source conductors. The results agree well with those of the conformal mapped model of Shercliff for a quadrupole source conductor problem. The method described here is straightforwardly applicable to problems where there is no hope of an analytic solution. For this reason, although it incorporates simplifying assumptions, the method is potentially quite useful as a means for experimenting with source conductor configurations so as to achieve desired liquid column geometries. The methods described in this paper could easily be applied to axisymmetric systems by solving the analogous axisymmetric fields problem and adding another term $\gamma / R(z)$ to the discrete surface tension term in Eq. (5) since the axisymmetric problems involves two local radii of curvature.

\section{Acknowledgments}

The authors gratefully acknowledge the help of Prof. Z. Cendes in the development of the tools described in this paper. His finite element and mesh generation code form essential ingredients in the work described here.

The authors also acknowledge valuable discussion with Prof. W. Hughes in the context of fluid mechanics, Prof. R. Fruehan and Prof. A. Cramb in the context of steelmaking, and Mr. A. Butler, Mr. B. Shyamkumar, Dr. D. Shenton and Dr. P. Levin in the context of numerical modeling.

This work was supported by the Center for Iron and Steelmaking Research (CISR) at CarnegieMellon University. Discussions with representatives of several CISR member companies have been quite 
useful in establishing the practical implications and usefulness of this work.

\section{REFERENCES}

1) Committee on Technology: Tech. Rep., "Continuous Casting of Steel 1985-A Second Study", Int. Iron and Steel Inst., Brussels, (1986).

2) W. A. Peifer: J. Met., (1965), May, 487.

3) J. Etay and M. Garnier: Metallurgical Applications of Magnetohydrodynamics, Proc. of Symp. of the Int. Union of Theoretical and $\Lambda$ pplied Mechanics, ed. by H. K. Moffat and M.R.E. Proctor, Met. Soc., London, (1982), 190.

4) S. G. Sagardia: " Electromagnetic Levitation Melting of Large Conductive Loads", Ph.D. thesis, University of Toronto, (1974).

5) J. A. Shercliff: Proc. R. Soc. (London), A375 (1981), 455.

6) A. Gagnoud, J. Etay and M. Garnier: Trans. Iron Steel Inst.
Jpn., 28 (1988), 36.

7) K. H. Huebner: The Finite Element Method for Engineers, John Wiley \& Sons, New York, (1975).

8) O. G. Zienkiewicz: The Finite Element Method, McGraw-Hill, London, (1977).

9) E. B. Becker, G. F. Carey and J. T. Oden: Finite Elements, I, Prentice-Hall, Englewood Cliffs, NJ, (1981).

10) P. P. Silvester and R. L. Ferrari: Finite Elements for Electrical Engineers, Cambridge Univ. Press, New York, (1983).

11) M.V.K. Chari and P.P. Silvester: Finite Elements in Electrical and Magnetic Field Problems, John Wiley \& Sons, New York, (1980).

12) Z.J. Cendes, D. Shenton and H. Shahnasser: IEEE Trans. Magn., MAG-19 (1983), 2551.

13) D. W. Fugate: "Computer modeling for magnetic confinement and shaping of liquid metals", Master's Thesis, Carnegie-Mellon University, PA, (1987). 